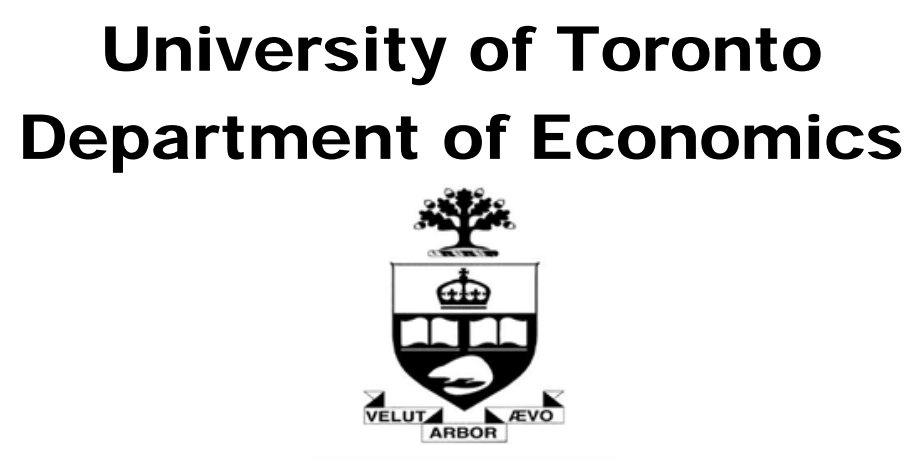

Working Paper 379

A Dynamic Game of Airline Network Competition: Hub-and-Spoke Networks and Entry Deterrence

By Victor Aguirregabiria and Chun-Yu Ho

October 28, 2009 


\title{
A Dynamic Game of Airline Network Competition: Hub-and-Spoke Networks and Entry Deterrence
}

\author{
Victor Aguirregabiria \\ University of Toronto
}

\author{
Chun-Yu Ho \\ Georgia Institute of Technology
}

October 1, 2009

\begin{abstract}
In a hub-and-spoke network, the total profit function of an airline is supermodular with respect to its entry decisions at different city-pairs. This source of complementarity implies that a hub-and-spoke network can be an effective strategy to deter entry of competitors. This paper presents a dynamic game of airlines network competition that incorporates this entry deterrence motive for using hub-and-spoke networks. We summarize the results of the estimation of the model, with particular attention to empirical evidence on the entry deterrence motive.
\end{abstract}

Keywords: Airline networks; Hub-and-spoke; Entry deterrence; Dynamic games; Supermodularity

\section{Corresponding Author:}

Victor Aguirregabiria

150 St. George Street

Toronto, ON, M5S 3G7

Canada

Phone: (416) 978-4358

E-mail: victor.aguirregabiria@utoronto.ca 


\section{Introduction}

An airline's network is a description of the city-pairs that the airline connects with non-stop flights. Two network structures that have received particular attention in studies of the airline industry are hub-and-spoke networks and point-to-point networks. In a hub-and-spoke network an airline concentrates most of its operations in one airport, called the hub. All other cities in the network (i.e., the spokes) are connected to the hub by non-stop flights such that travellers between two spoke-cities should take a connecting flight at the hub. In contrast, in a point-to-point network all cities are connected with each other through nonstop flights. Pure hub-and-spoke or pure point-to-point networks are very rear. Most airlines have some degree of 'hubbing'. Table 1 presents measures of "hubbing" based on airlines' networks in the 55 largest US cities. These measures try to capture the degree in which an airline's operation is concentrated in one or a few airports. ${ }^{1}$

The choice of network structure is one of the most important strategic decisions of an airline. The relationship between network structure and airlines' operating costs has received significant attention by IO economists, both in theoretical and in empirical work. Different studies have shown how a hub-and-spoke network can exploit significant economies of scope at the airport level and economies of traffic density. ${ }^{2}$

Other aspect of airline network competition that has received theoretical attention is the airlines' use of hub-and-spoke network as a strategy to deter the entry of competitors. This argument was first established by Hendricks, Piccione and Tan (1997) using a three-stage

\footnotetext{
${ }^{1}$ Table 1 presents two statistics, $C R 1$ and $C R 2$. Let $n$ be the total number of non-stop connections of an airline. Let $n^{(1)}$ be the number of non-stop connections of the airline at the airport where it has more connections. Similarly, let $n^{(k)}$ be the number of non-stop connections of the airline in its k-th largest airport, excluding connections with any of its k-1 largest airports, if those connections exist. Then, for any inter $K$ greater or equal than one, the index $C R K$ is defined as $\left(\sum_{k=1}^{K} n_{i}^{(k)}\right) / n_{i}$. For a pure hub-and-spoke network, $C R 1=1$. For a network with two hubs, we have that $C R 1>1 / 2$ and $C R 2=1$. For a pure point-to-point network, $C R 1=2 / n$.

${ }^{2} \mathrm{~A}$ hub-and-spoke network can fully connect $C$ cities using the minimum number of direct connections, $C-1$. Therefore, it minimizes fixed costs associated with establishing non-stop connections. Furthermore, the different routes that an airline has in the same airport may share some common operating costs. An airline can benefit from these economies of scope by concentrating their operation in a few airports. Last, but not least, larger planes are more efficient on a per-seat basis and airlines can exploit these cost savings by seating in a single plane passengers who have different final destinations or who come from different origins (i.e., economies of traffic density). See Caves, Christensen, and Tretheway (1984), Berry (1990), Brueckner and Spiller (1991), and Brueckner (2004), among others.
} 
sequential game of entry between an incumbent hub-and-spoke carrier and a point-to-point regional carrier. ${ }^{3}$ The profit function of a hub-and-spoke airline is supermodular with respect to its entry decisions at different city-pairs. This complementarity implies that a hub-andspoke airline may be willing to operate non-stop flights in a city-pair even when profits are negative because operation in that city-pair can generate positive profits in other routes. This is known by potential entrants, and therefore entry may be deterred. This argument for entry deterrence does not suffer from several limitations of other more standard arguments of predatory conduct. In particular, it does not require of a sacrifice of the incumbent (i.e., reduction in current profits) that will be compensated in the future only if competitors do not enter in the market. ${ }^{4}$ Also, it is not subject to Judd's criticism to arguments of spatial entry deterrence (Judd, 1985). ${ }^{5}$

Despite these attractive features of Hendricks-Piccione-Tan entry deterrence argument, there are not previous studies that explore empirically this entry deterrence motive in airline use of hub-and-spoke networks. Part of the reason for this lack of empirical evidence is the absence of structural models of dynamic network competition that incorporate this hypothesis and that are flexible and realistic enough to be estimated with actual data. The objective of this paper is to present a dynamic game of airlines network competition that incorporates the strategic entry deterrence motive of a hub-and-spoke network and that can be estimated using publicly available data from the US Bureau of Transportation. Also, we describe how the estimated model can be used to test for strategic entry deterrence. In a companion

\footnotetext{
${ }^{3}$ See also Oum, Zhang, and Zhang (1995) and Hendricks, Piccione and Tan (1999).

${ }^{4}$ It is difficult to generate this type of predatory conduct as a stationary Markov perfect equilibrium. Furthermore, in antitrust cases, it is typically quite difficult to find convincing empirical evidence on the sacrifice component of the argument. See the papers by Kim (2009) and Snider (2009) that deal with this issue in US vs. American Airlines case.

${ }^{5}$ Judd (1985) notes that some models of entry and spatial location that generate entry deterrence as a subgame perfect equilibrium place strong assumptions on firms' level of commitment. Those papers assume that entry and location decisions are completely irreversible, with no possibility of exit or relocation. Judd shows that when there is strong enough substitutability among the stores of the same firm, allowing for exit may result in non-successful spatial preemption by the incumbent. Potential entrants know the incumbent firm may prefer to have a monopoly in a single location rather than being a monopolist in a location and a duopolist in another nearby location. Therefore, spatial preemption and entry deterrence by the incumbent is not a credible strategy. This type of argument does not apply to a hub-and-spoke airline because profits at different city-pairs (different "stores") are not substitute but complements. This complementarity makes entry-deterrence a credible strategy in equilibrium.
} 
paper (Aguirregabiria and Ho, 2009), we estimate this model and use it to measure the contribution of demand, cost, and strategic factors to explain hub-and-spoke networks. We summarize here the main empirical results of that paper, with particular attention to the empirical evidence on the entry deterrence motive.

\section{Model}

The industry is configured by $N$ airline companies and $C$ cities. A market is a city-pair. Entry/exit in a city-pair is not directional, i.e., if an airline operates flights from city $A$ to city $B$, then it should operate flights from $B$ to $A$. Therefore, there are $M \equiv C(C-1) / 2$ markets or city-pairs. We index time by $t$, markets by $m$, and airlines by $i$. The network of an airline consists of the set of city-pairs in which the airline operates non-stop flights. It can be represented by a vector $\mathbf{x}_{i t} \equiv\left\{x_{i m t}: m=1,2, \ldots, M\right\}$, where $x_{i m t} \in\{0,1\}$ is the binary indicator of the event "airline $i$ operates non-stop flights in city-pair $m$ at period $t$ ". The whole industry network is represented by the vector $\mathbf{x}_{t} \equiv\left\{\mathbf{x}_{i t}: i=1,2, \ldots, N\right\} \in \mathcal{X}$, where $\mathcal{X} \equiv\{0,1\}^{N M}$. Travellers are concerned about routes. A route is a directional roundtrip between two cities. A network describes implicitly all the routes for which an airline provides flights, either stop or non-stop. In principle, we can construct routes with many (even infinite) stops. We consider only routes with zero, one, or two stops. ${ }^{6} L\left(\mathbf{x}_{i t}\right)$ is the set of these routes associated with network $\mathbf{x}_{i t}$. We index routes by $r$.

Every period (quarter) ${ }^{7} t$, airlines take as given the current industry network $\mathbf{x}_{t}$ and choose prices for all the routes where they operate flights, either non-stop or stop. Price competition (static $)^{8}$ determines variable profits for each airline and route. Airlines also decide their networks for next quarter, $\mathbf{x}_{i t+1}$. There is time-to-build such that fixed costs and entry costs are paid at quarter $t$ but entry-exit decisions are not effective until quarter

\footnotetext{
${ }^{6}$ Routes with more than two stops represent less than $1 \%$ of all the air tickets in the US Origin and Destination (DB1B) database.

${ }^{7}$ The DB1B database has quarterly frequency.

${ }^{8}$ Intertemporal price discrimination and plane capacity constraints can generate dynamic (forwardlooking) pricing strategies at the level of individual flights (i.e., specific flight number and day). However, that type of pricing dynamics is short-run and flight-specific and it plays a very minor role in the dynamics of network structure. For simplicity, this model ignores dynamic pricing.
} 
$t+1$. The airline's total profit function is:

$$
\Pi_{i t}=\sum_{r \in L\left(\mathbf{x}_{i t}\right)} R_{i r}\left(\mathbf{x}_{t}\right)-\sum_{m=1}^{M} x_{i m t+1}\left(F C_{i m t}+\left(1-x_{i m t}\right) S C_{i m t}\right)
$$

$R_{i r}\left(\mathbf{x}_{t}\right)$ is the variable profit of airline $i$ in the Nash-Bertrand equilibrium for route $r . F C_{i m t}$ represents the fixed cost for airline $i$ in market $m$ and quarter $t$. And $S C_{i m t}$ is a start-up cost or entry cost at the city-pair level, i.e., an additional fixed cost that should be paid if airline $i$ was not active in market $m$ at period $t$ and it decides to start operating at period $t+1$. The equilibrium of the dynamic game implies a Markov transition probability for the industry network, $\operatorname{Pr}\left(\mathbf{x}_{t+1} \mid \mathbf{x}_{t}\right)$, and its corresponding ergodic probability distribution, $p^{*}\left(\mathbf{x}_{t}\right) .^{9}$

Consumer demand and price competition. This part of the framework is a standard logit model of demand and price competition. For notational simplicity, we omit the time subindex $t$ in the description of this static demand model. Let $H_{r}$ be the number of potential travelers in route $r$. For a given route $(r)$, there are two forms of product differentiation: the airline $(i)$, and the indicator of non-stop flight $(N S)$. Travelers decide which product $(i, N S)$ to purchase, if any. The indirect utility of a consumer in route $r$ who purchases product $(i, N S)$ is $b_{i, r, N S}-p_{i, r, N S}+v_{i, r, N S}$, where $p_{i, r, N S}$ is the price, $b_{i, r, N S}$ is the quality of the product, and $v_{i, r, N S}$ is a consumer-specific component that captures consumer heterogeneity in preferences. Travellers can choose an outside alternative of not travelling by air. Quality and price of the outside alternative are normalized to zero. In this paper, we ignore hubsize effects on demand and variable costs and consider a simple specification for product quality: ${ }^{10} b_{i, r, N S}=\alpha_{i}^{(0)}+\alpha_{i}^{(1)} N S$, where $\alpha_{i}^{(0)}$ is the quality of an stop-flight, and $\alpha_{i}^{(0)}+\alpha_{i}^{(1)}$ is the quality of a nonstop-flight of airline $i$. We assume that $v_{i, r, N S}$ are independent Type I extreme value random variables. Therefore, the aggregate demand of product $(i, N S)$ in route $r$ is $q_{i, r, N S}=\exp \left\{b_{i, r, N S}-p_{i, r, N S}\right\} /\left[1+\sum_{k} \exp \left\{b_{k}-p_{k}\right\}\right]$, where the sum $\sum_{k}$ is over all the products available for route $r$. The variable profit of airline $i$ in route $r$ is

\footnotetext{
${ }^{9}$ In a more general version of the model that includes exogenous state variables affecting demand and costs, that we represent with the vector $\mathbf{z}_{t}$, the dynamics of the industry can be described using the endogenous Markov transition probability $\operatorname{Pr}\left(\mathbf{x}_{t+1} \mid \mathbf{x}_{t}, \mathbf{z}_{t}\right)$ and the exogenous transition probability of $\mathbf{z}_{t}$.

${ }^{10}$ Aguirregabiria and Ho (2009) consider a richer specification of demand and variable costs that includes a nested logit structure for travellers' idiosyncratic preferences, permanent airline and city heterogeneity, and hub-size effects in both demand and variable costs.
} 
$R_{i r}=\left(p_{i r 0}-c_{i r 0}\right) q_{i r 0}+\left(p_{i r 1}-c_{i r 1}\right) q_{i r 1}$, where $c_{i, r, N S}$ is the constant marginal cost of product $(i, r, N S)$. The specification of this marginal cost is similar to the one of product quality: $c_{i, r, N S}=\omega_{i}^{(0)}+\omega_{i}^{(1)} N S$, where $\omega_{i}^{(0)}$ and $\omega_{i}^{(0)}+\omega_{i}^{(1)}$ represent the marginal costs of stop-flights and non-stop flights for airline $i$, respectively. Equilibrium prices depend on the qualities and marginal costs of all the airlines and products that are active in the same route.

For an airline with a hub-and-spoke network, the (total) variable profit function $T R_{i} \equiv$ $\sum_{r \in L\left(\mathbf{x}_{i}\right)} R_{i r}(\mathbf{x})$ is supermodular with respect to the airline entry decisions at different citypairs. Consider an airline with hub at city $A$ and spoke cities $B$ and $C$. The total variable profit is $T R_{i}\left(x_{i, A B}, x_{i, A C}\right)=x_{i, A B} R_{i, A B}+x_{i, A C} R_{i, A C}+x_{i, A B} x_{i, A C} R_{i, B C}$. If the airline operates in city-pair $A C$, the additional profit of operating in city-pair $A B$ is not only $R_{i, A B}$ but $R_{i, A B}+R_{i, B C}$. Therefore, if $R_{i, B C}>0$, there is complementarity between the decisions of operating in city-pairs $A B$ and $A C$. Or more formally, the airline's variable profit function is supermodular in $\left(x_{i, A B}, x_{i, A C}\right)$, i.e., $\left[T R_{i}(1,1)-T R_{i}(0,1)\right]-\left[T R_{i}(1,0)-T R_{i}(0,0)\right]=R_{i, B C}>$ 0.

Fixed Costs and Start-Up Costs. ${ }^{11}$ The structure of the fixed cost $F C_{i m t}$ is $F C_{i m t}=\left(\gamma_{i}^{F C(0)}-\right.$ $\left.\gamma_{i}^{F C(1)} H U B_{i m t}\right)+\varepsilon_{i m t}^{F C}$, where $\gamma_{i}^{F C(0)} \geq 0$ and $\gamma_{i}^{F C(1)} \geq 0$ are parameters. The component $\varepsilon_{i m t}^{F C}$ is private information of the airline. This private information shock is assumed to be independently and identically distributed over firms and over time with zero mean. $H U B_{i m t}$ represents the hub-size of airline $i$ in the airports of city-pair $m$ as measured by the total number of cities that airline $i$ connects with nonstop-flights from the origin and destination airports in city-pair $m$ : $H U B_{i m t} \equiv \sum_{m^{\prime} \in \mathcal{C}_{m}} x_{i m^{\prime} t}$, where $\mathcal{C}_{m}$ is the set of markets with a common city with market $m$. The parameter $\gamma_{i}^{F C(0)}$ represents airline $i$ 's fixed cost in a market where it does not have any other connections. The parameter $\gamma_{i}^{F C(1)}$ measures how airline $i$ 's fixed cost declines with its hub-size in the city-pair. The start-up cost $S C_{i m t}$ has the same structure as the fixed cost: $S C_{i m t}=\left(\delta_{i}^{S C(0)}-\delta_{i}^{S C(1)} H U B_{i m t}\right)+\varepsilon_{i m t}^{S C}$, where $\delta_{i}^{S C(0)} \geq 0$ and $\delta_{i}^{S C(1)} \geq 0$ are parameters.

When $\gamma_{i}^{F C(1)}$ or $\delta_{i}^{S C(1)}$ are strictly positive, profits at different city-pairs are interconnected

\footnotetext{
${ }^{11} \mathrm{As}$ in the case of variable profits, Aguirregabiria and Ho (2009) consider a richer specification of fixed costs and entry costs that includes both airline and city permanent heterogeneity.
} 
through the hub-size effects. This is a second source of complementarity between an airline's entry decisions at different city-pairs.

Markov Perfect Equilibrium (MPE). Airlines maximize intertemporal profits. They are forward-looking and take into account the implications of their entry-exit decisions on future profits and on the expected future reaction of competitors. We assume that airlines' strategies depend only on payoff-relevant state variables, i.e., Markov perfect equilibrium assumption. An airline's payoff-relevant information at quarter $t$ is $\left\{\mathbf{x}_{t}, \boldsymbol{\varepsilon}_{i t}\right\}$, where $\boldsymbol{\varepsilon}_{i t}$ is the vector of airline and market-specific private information shocks $\left\{\varepsilon_{i m t}^{F C}, \varepsilon_{i m t}^{S C}: m=1,2, \ldots, M\right\}$. Let $\boldsymbol{\sigma} \equiv\left\{\sigma_{i}\left(\mathbf{x}_{t}, \boldsymbol{\varepsilon}_{i t}\right): i=1,2, \ldots, N\right\}$ be a set of strategy functions, one for each airline, such that $\sigma_{i}$ is a function from $\mathcal{X} \times \mathbb{R}^{2 M}$ into $\{0,1\}^{M}$. A MPE in this game is a set of strategy functions such that each airline's strategy maximizes its value for each possible state $\left(\mathbf{x}_{t}, \boldsymbol{\varepsilon}_{i t}\right)$ and taking as given other airlines' strategies.

Let $V_{i}^{\sigma}\left(\mathbf{x}_{t}, \varepsilon_{i t}\right)$ represent the value function for airline $i$ given that the other airlines behave according to their respective strategies in $\boldsymbol{\sigma}$, and given that airline $i$ uses his best response/strategy. By the principle of optimality, this value function is implicitly defined as the unique solution to the following Bellman equation:

$$
V_{i}^{\sigma}\left(\mathbf{x}_{t}, \boldsymbol{\varepsilon}_{i t}\right)=\max _{\mathbf{x}_{i t+1}}\left\{\Pi_{i}\left(\mathbf{x}_{t}, \mathbf{x}_{i t+1}\right)-\varepsilon_{i t}\left(\mathbf{x}_{i t+1}\right)+\beta E\left[V_{i}^{\sigma}\left(\mathbf{x}_{t+1}, \boldsymbol{\varepsilon}_{i t+1}\right) \mid \mathbf{x}_{t}, \mathbf{x}_{i t+1}\right]\right\}
$$

where $\beta \in(0,1)$ is the discount factor; $\Pi_{i}\left(\mathbf{x}_{t}, \mathbf{x}_{i t+1}\right)$ represents the common-knowledge part of the profit function, i.e., $\Pi_{i}\left(\mathbf{x}_{t}, \mathbf{x}_{i t+1}\right) \equiv \sum_{r \in L\left(\mathbf{x}_{i t}\right)} R_{i r}\left(\mathbf{x}_{t}\right)-\sum_{m=1}^{M} x_{i m t+1}\left(\left(\gamma_{i}^{F C(0)}-\right.\right.$ $\left.\left.\gamma_{i}^{F C(1)} H U B_{i m t}\right)+\left(1-x_{i m t}\right)\left(\delta_{i}^{S C(0)}-\delta_{i}^{S C(1)} H U B_{i m t}\right)\right)$; and the term $\varepsilon_{i t}\left(\mathbf{x}_{i t+1}\right)$ contains the private information part of the profit function, i.e., $\varepsilon_{i t}\left(\mathbf{x}_{i t+1}\right) \equiv \sum_{m=1}^{M} x_{i m t+1}\left(\varepsilon_{i m t}^{F C}+(1-\right.$ $\left.\left.x_{i m t}\right) \varepsilon_{i m t}^{S C}\right)$. A set of strategies $\boldsymbol{\sigma}$ is a MPE if, for every airline $i$ and every state $\left(\mathbf{x}_{t}, \boldsymbol{\varepsilon}_{i t}\right)$, we have that:

$$
\sigma_{i}\left(\mathbf{x}_{t}, \boldsymbol{\varepsilon}_{i t}\right)=\arg \max _{\mathbf{x}_{i t+1}}\left\{\Pi_{i}\left(\mathbf{x}_{t}, \mathbf{x}_{i t+1}\right)-\varepsilon_{i t}\left(\mathbf{x}_{i t+1}\right)+\beta E\left[V_{i}^{\sigma}\left(\mathbf{x}_{t+1}, \boldsymbol{\varepsilon}_{i t+1}\right) \mid \mathbf{x}_{t}, \mathbf{x}_{i t+1}\right]\right\}
$$

That is, every airline is in its best response to the other airlines' strategies. An equilibrium in this dynamic game provides a description of the joint dynamics of prices, quantities, and airlines' incumbent status for every route between the $C$ cities of the industry. 
For the computation of an equilibrium, and for the structural estimation of the model, it is convenient to define a MPE in terms of airlines' conditional choice probabilities (CCPs). Define the choice probability $P_{i}\left(\mathbf{x}_{i t+1} \mid \mathbf{x}_{t}\right) \equiv \int 1\left\{\sigma_{i}\left(\mathbf{x}_{t}, \boldsymbol{\varepsilon}_{i t}\right)=\mathbf{x}_{i t+1}\right\} d G_{\varepsilon}\left(\varepsilon_{i t}\right)$, where $1\{$.$\} is$ the indicator function, and $G_{\varepsilon}$ is the CDF of $\varepsilon_{i t} . P_{i}\left(\mathbf{x}_{i t+1} \mid \mathbf{x}_{t}\right)$ is the probability that airline $i$ operates a network $\mathbf{x}_{i t+1}$ at period $t+1$ given that the industry network at period $t$ is $\mathbf{x}_{t}$. Let $\mathbf{P}$ be the vector of CCPs associated with $\boldsymbol{\sigma}$, i.e., $\mathbf{P}=\left\{P_{i}\left(\mathbf{x}_{i t+1} \mid \mathbf{x}_{t}\right): i=1,2, \ldots, N\right.$; $\left.\mathbf{x}_{i t+1} \in\{0,1\}^{M} ; \mathbf{x}_{t} \in \mathcal{X}\right\}$. Following Aguirregabiria and Mira (2007), a MPE in this dynamic game can be described as a vector of probabilities $\mathbf{P}$ that solves the fixed point problem $\mathbf{P}=\Psi(\mathbf{P})$, where $\Psi(\mathbf{P})$ is a vector-valued best-response probability function.

Estimation. The vector of structural parameters of the model, $\boldsymbol{\theta}$, includes parameters in demand, $\left\{\alpha_{i}^{(0)}, \alpha_{i}^{(1)}\right\}$, variable costs, $\left\{\omega_{i}^{(0)}, \omega_{i}^{(1)}\right\}$, fixed costs, $\left\{\gamma_{i}^{F C(0)}, \gamma_{i}^{F C(1)}\right\}$, and entry costs, $\left\{\delta_{i}^{S C(0)}, \delta_{i}^{S C(1)}\right\}$. Aguirregabiria and Ho (2009) show that these parameters are identified using data on prices and quantities at the airline-route level (for the identification of demand and variable cost parameters), and on the transition of airlines' networks (for the identification of fixed costs and entry costs).

Entry Deterrence and Hub-and-Spoke Networks. Given consistent estimates of the vector of structural parameters $\boldsymbol{\theta}$, and of the equilibrium in the data, as represented by the vector of choice probabilities $\mathbf{P}$, we are interested in measuring the role of hub-and-spoke networks as a credible strategy to deter entry of point-to-point carriers. This entry deterrence argument is based on the supermodularity (complementarity) of the total variable profit function of a hub-and-spoke airline. The elimination of this supermodularity should also remove this potential source of entry deterrence. More specifically, if this supermodularity generates entry deterrence, then by eliminating it for a certain airline we should find that the airline has both a lower propensity to 'hubbing' (i.e., lower concentration of its operation in a few cities as measured by concentration ratios $C R 1$ or $C R 2$ ), and a lower number of city-pairs where it operates as a monopolist.

To implement this type of comparative statics exercise, we need to define a counterfactual scenario where we eliminate this source supermodularity in the variable profit of a hub-andspoke airline. We consider the following approach. Suppose that we describe an airline as 
a group of local managers, one for each city-pair $m$. The double index $(i, m)$ represents the local manager of airline $i$ in market $m$. This local manager decides whether to operate non-stop flights in city-pair $m$. In our model, the decision making of an airline is centralized. Therefore, the model assumes that all the local managers of an airline internalize the complementarities between their entry-exit decisions. ${ }^{12}$ To eliminate supermodularity in an airline variable profits we consider the counterfactual scenario where the local managers of an airline are concerned with the maximization of its own city-pair profit that includes only the variable profit from non-stop flights between the two cities. This counterfactual local manager ignores that his city-pair is a segment of many other routes and that the operation of his city-pair can generate additional profits in these other routes. For instance, consider the example of the hub-and-spoke airline with hub at city $A$ and spokes at cities $B$ and $C$. The total variable profit of the airline is $T R\left(x_{A B}, x_{A C}\right)=x_{A B} R_{A B}+x_{A C} R_{A C}+x_{A B} x_{A C} R_{B C}$, that is a supermodular function when $R_{B C}>0$. However, in the counterfactual scenario, the local manager in city pair $A B$ is only concerned with its local variable profit $x_{A B} R_{A B}$ (and the local manager $A C$ is only concerned with profit $\left.x_{A C} R_{A C}\right)$. Therefore, these "uncoordinated" local managers do not take into account the complementarity of their decisions in the total profit of the airline. In an equilibrium of this counterfactual model, we expect that the network of the airline presents a lower degree of 'hubbing'. Furthermore, if the entry deterrence motive is significant in the factual equilibrium, we expect that in the counterfactual the airline will be a monopolist in a smaller number of markets.

\section{$3 \quad$ Empirical evidence}

Aguirregabiria and Ho (2009) estimate the dynamic game that we have described above using data from the Airline Origin and Destination Survey (DB1B) for the 55 largest cities in US. Here we summarize the main empirical results in that paper, with particular attention to those results related to strategic entry deterrence.

\footnotetext{
${ }^{12}$ The estimated model in Aguirregabiria and Mira (2009) considers a certain degree of decentralization in local managers decision making. However, in that model, local managers still internalize the complementarities in their operation decisions.
} 
Economies of scope in fixed costs and start-up costs. Estimated fixed costs and start-up costs at the city-pair level are sizeable. The mean value (averaged over airlines and markets) of fixed costs is $\$ 119,000$ or $75 \%$ of city-pair quarterly variable profit. Start-up costs are on average equal to $\$ 298,000$, that accounts for $187 \%$ of city-pair quarterly variable profit. Although we find very important airline heterogeneity in estimated fixed and start-up costs, the average airline has significant incentives to save these costs by reducing the number of non-stop connections using a hub-and-spoke network. These incentives are even larger when we take into account economies of scope at the airport level. The estimated values of the parameters that measure these economies of scope are $\gamma^{F C(1)}=\$ 1,020$ and $\delta^{S C(1)}=\$ 9,260$. That is, one additional non-stop connection (i.e., a unit increase in hub-size $H U B_{i m t}$ ) reduces city-pair fixed costs in $\$ 1,020$ and start-up costs in $\$ 9,260$. Counterfactual experiments show that these economies of scope in fixed costs and, particularly, in start-up costs play an important role to explain the propensity of legacy carriers to use hub-and-spoke networks.

Strategic entry deterrence. To measure the entry deterrence motive of hub-and-spoke networks, we implement the counterfactual experiment described at the end of previous section. ${ }^{13}$ Table 2 presents a summary of the counterfactual experiments. Each row represents a different experiment, i.e., a experiment where we eliminate supermodularity in the variable profit of a single airline, leaving the other airlines unchanged, and calculate a new equilibrium of the dynamic game.

For all the legacy airlines (i.e., all the airlines in Table 2 except Southwest), eliminating supermodularity in variable profits implies a significant reduction in the concentration ratio $C R 2$ that measures the degree of 'hubbing'. Interestingly, there is also an important reduction in the number of city-pairs where the airline operates as a monopolist. ${ }^{14}$ The reduction in $C R 2$ and in the number of monopoly markets is particularly important for Northwest and Delta. It seems that the entry deterrence motive of using a hub-and-spoke network plays an

\footnotetext{
${ }^{13}$ To deal with the problem of multiple equilibria under the counterfactual scenario, we implement the method in Aguirregabiria (2009).

${ }^{14}$ We should clarify our use of the term monopolist in Table 2 . What we mean is that the airline is the only carrier that operates non-stop flights in the city-pair. Of course, this does not mean that the airline is really a monopolist in the routes between the two cities because there may be other airlines that operate stop flights that connect the two cities. Nevertheless, given that consumers prefer non-stop flights to stop flights, it is clear that being the only carrier operating non-stop flights provides significant market power.
} 
important role for these airlines. Northwest and Delta are the airlines that, after Southwest, operate as monopolists in a larger number of city-pairs and that have largest hub sizes. Also, these airlines tend to operate as monopolies in city-pairs with relatively small market size. According to our estimates, the large number of monopoly markets enjoyed by these carriers cannot be explained by more efficient exogenous costs, i.e., by lower values of the parameters $\omega_{i}^{(0)}, \omega_{i}^{(1)}, \gamma_{i}^{F C(0)}$, or $\delta_{i}^{S C(0)}$. Instead, it is mainly explained by lower values of the endogenous part of the costs, i.e., $-\gamma_{i}^{F C(1)} H U B_{i m}$ and $-\delta_{i}^{S C(1)} H U B_{i m}$.

In a certain sense, Southwest represents the opposite case to Northwest and Delta. Southwest is, by far, the airline with the smallest contribution of the entry deterrence motive. According to the estimated model, Southwest enjoys a large number of monopoly markets because it has much lower values than any other airline for the exogenous part of the cost $\left(\omega_{i}^{(0)}, \omega_{i}^{(1)}, \gamma_{i}^{F C(0)}\right.$, and $\left.\delta_{i}^{S C(0)}\right)$. This cost efficiency allows the airline to operate in small markets. Other interesting result of our experiments is that in the counterfactual equilibrium, Southwest tends to enter in a large proportion of the markets that Northwest and Delta leave when we eliminate supermodularity in their profit functions. Again, this result is consistent with an entry deterrence use of their hub-and-spoke networks by Northwest and Delta.

Nevertheless, the net welfare effect of this type of entry deterrence behavior is ambiguous. On the one hand, this strategy is restricting the entry of carriers that have ex-ante lower operating costs, such as Southwest Airlines. On the other hand, the estimated model shows that hub-and-spoke networks exploit economies of scope and density that generate ex-post very significant cost reductions. Southwest low-cost strategy has shown to be an effective way to compete with large hub-and-spoke carriers. However, the estimated model shows that this does not mean that the Pareto optimal structure of the industry should be one with many "Southwest-like" point-to-point carriers. This industry structure would not exploit very significant cost savings associated with hub-and-spoke networks. In this context, it is of great interest the consideration and evaluation of policies that try to reduce the entry deterrence effect of hub-and-spoke networks but that can maintain most of the cost savings associated with these networks. 


\section{References}

[1] Aguirregabiria, V., and C-Y Ho (2009): "A Dynamic Oligopoly Game of the US Airline Industry: Estimation and Policy Experiments," manuscript. Department of Economics. University of Toronto.

[2] Aguirregabiria, V., and P. Mira (2007): "Sequential Estimation of Dynamic Discrete Games," Econometrica, 75, 1-53.

[3] Aguirregabiria, V. (2009): "A Method for Implementing Counterfactual Experiments in Models with Multiple Equilibria," manuscript. Department of Economics. University of Toronto.

[4] Berry, S. (1990): "Airport Presence as Product Differentiation," American Economic Review, 80, 394-399.

[5] Brueckner, J. (2004): "Network Structure and Airline Scheduling," Journal of Industrial Economics, 52, 291-312.

[6] Brueckner, J., and P. Spiller (1991): "Competition and Mergers in Airline Networks," International Journal of Industrial Organization, 9, 323-342.

[7] Caves, D., L. Christensen, and M. Tretheway (1984): "Economies of Density Versus Economies of Scale: Why Trunk and Local Service Airline Costs Differ," Rand Journal of Economics, 15, 471-489.

[8] Hendricks, K., M. Piccione, and G. Tan (1997): "Entry and exit in hub-spoke networks," Rand Journal of Economics, 28(2), 291-303.

[9] Hendricks, K., M. Piccione, and G. Tan (1999): "Equilibria in Networks," Econometrica, 67, 1407-1434.

[10] Judd, K. (1985): "Credible Spatial Preemption," The RAND Journal of Economics, 16(2), 153-166.

[11] Kim, S-H (2009): "Predatory Reputation in US Airline Markets," International Journal of Industrial Organization, 27, 592-604.

[12] Oum, T., A. Zhang, and Y. Zhang (1995): "Airline Network Rivalry," Canadian Journal of Economics, 28, 836-857.

[13] Snider, C. (2009): "Predatory Incentives and Predation Policy: The American Airlines Case," manuscript. Department of Economics. UCLA. 


\begin{tabular}{|c|c|c|c|c|}
\hline \multicolumn{5}{|c|}{$\begin{array}{c}\text { Table 1 } \\
\text { Measures of 'Hubbing' in the US Airline Industry: Year } 2004\end{array}$} \\
\hline Airline (Code) & $\begin{array}{l}\text { 1st largest hub } \\
\text { (\# connections) }\end{array}$ & $\begin{array}{l}\text { Concentration } \\
\text { Ratio CR1 }\end{array}$ & $\begin{array}{l}\text { 2nd largest hub } \\
\text { (\# connections) }\end{array}$ & $\begin{array}{l}\text { Concentration } \\
\text { Ratio CR2 }\end{array}$ \\
\hline Southwest (WN) & Las Vegas (35) & 9.3 & Phoenix (33) & 18.2 \\
\hline American (AA) & Dallas (52) & 22.3 & Chicago (46) & 42.0 \\
\hline United (UA) & Chicago $(50)$ & 25.1 & Denver (41) & 45.7 \\
\hline Delta (DL) & Atlanta (53) & 26.7 & Cincinnati (42) & 48.0 \\
\hline Continental (CO) & Houston (52) & 36.6 & New York $(45)$ & 68.3 \\
\hline Northwest (NW) & Minneapolis (47) & 25.6 & Detroit (43) & 49.2 \\
\hline US Airways (US) & Charlotte (35) & 23.3 & Philadelphia (33) & 45.3 \\
\hline America West (HP) & Phoenix (40) & 35.4 & Las Vegas (28) & 60.2 \\
\hline Alaska (AS) & Seattle (18) & 56.2 & Portland (10) & 87.5 \\
\hline ATA (TZ) & Chicago (16) & 48.4 & Indianapolis (6) & 66.6 \\
\hline JetBlue (B6) & New York (13) & 59.0 & Long Beach (4) & 77.3 \\
\hline Frontier (F9) & Denver (27) & 56.2 & Los Angeles (5) & 66.6 \\
\hline AirTran (FL) & Atlanta (24) & 68.5 & Dallas (4) & 80.0 \\
\hline Trans States (AX) & St Louis (18) & 62.0 & Pittsburgh (7) & 93.9 \\
\hline Reno Air (QX) & Portland (8) & 53.3 & Denver $(7)$ & 100.0 \\
\hline Sun Country (SY) & Minneapolis (11) & 100.0 & (0) & 100.0 \\
\hline
\end{tabular}

Source: DB1B Database form the US Bureau of Transportation. Year 2004.

\section{Table 2}

\section{Entry Deterrence Motive of Hub-and-Spoke Networks}

\begin{tabular}{r|c|c|c|c}
\hline \multicolumn{1}{c|}{$\begin{array}{c}\text { Observed } \\
\text { Carrier }\end{array}$} & $\begin{array}{c}\text { Counterfactual } \\
\text { Concentration Ratio } \\
\text { CR2 }\end{array}$ & $\begin{array}{c}\text { Observed } \\
\text { Contration Ratio } \\
\text { CR2 }\end{array}$ & $\begin{array}{c}\text { Counterfactual } \\
\text { \# City-Pairs where } \\
\text { Airline is a Monopolist }\end{array}$ & $\begin{array}{c}\text { \# of City-Pairs where } \\
\text { Airline is a Monopolist }\end{array}$ \\
\hline Southwest & 18.2 & 16.5 & 151 & $149(-1 \%)$ \\
American & 42.0 & 24.5 & 31 & $26(-16 \%)$ \\
United & 45.7 & 30.3 & 16 & $12(-25 \%)$ \\
Delta & 48.0 & 22.1 & 57 & $21(-63 \%)$ \\
Continental & 68.3 & 42.8 & 27 & $20(-26 \%)$ \\
Northwest & 49.2 & 23.2 & 66 & $6(-62 \%)$ \\
US Airways & 45.3 & 35.2 & 8 & \\
\hline \hline
\end{tabular}

Based on estimation results in Aguirregabiria and Ho (2009) 